\title{
Fusing Vision and Range for Motion Estimation in Hardware
}

\author{
Julian Kolodko, Ljubo Valcic, Member, IEEE
}

\begin{abstract}
Obtaining and segmenting motion information in real time requires both an appropriate algorithm and a hardware implementation of that algorithm so that the massive video data stream is processed in a timely manner. This paper details an algorithm suitable for hardware implementation that provides motion information for autonomous vehicle navigation. This algorithm utilizes range information to disambiguate visual data. Simulation results illustrate the correct operation of our algorithm.
\end{abstract}

Index Terms - FPGA, Data Fusion, Motion Estimation, Motion Segmentation.

\section{INTRODUCTION}

Motion estimation using visual data has been studied in great detail [1] and a broad range of motion estimation techniques exist though these can be collected into three broad groups. The first group consists of the token-based methods (correlation [2] and feature tracking [3]) where image features are tracked between frames. The second group consists of methods based on frequency domain techniques [4]. Finally there are the gradient-based techniques that use image derivatives and are based on the optical flow constraint equation (OFCE) [5] or some variant [6]. These methods all operate solely on visual data and are susceptible to depth ambiguity where distant objects: appear to move across the image more slowly than nearby objects that have the same velocity.

We aim to overcome this ambiguity directly by fusing range and visual data. Our approach is similar to [7] though we a combination of range and visual data rather than visual and egomotion data. Further, we use a different statistical framework and aim to minimise processing complexity. Other methods of fusing range and visual data include the simultaneous minimisation of the optical and range flow equations [8], use of visual stereo [9] and the multiple model framework of [10] though these methods do not attempt to minimise computational complexity nor do they segment the environment in terms of moving objects.

Manuscript received April 30, 2003.

Julian Kolodko is with the Intelligent Control Systems Laboratory, School of Microclectronic Enginecring, Griffith University, Nathan QLD 4111, AUSTRALIA (phone: +61-7-3875-5024, c-mail: j.kolockonagriffill.cdu.au)

Ljubo Vlacic is with the Intelligent Control Systems Laboratory, School of Microelectronic Engincering, Griffith University, Nathan QLD 4111, AUSTRALIA (e-mail: l.vlaciciangriffith.cdu.au)
The idea of segmenting the environment in terms of moving regions is core to our work. We treat motion as a primitive visual sensory modality like brightness or colour. That is, we attempt to pull apart the image in terms of the independent motion of object fragments (i.e. "moving blobs"). No attempt is made to reconstruct the environment in terms of the objects that are moving. This approach is reasonable within the context of our work because it is not relevant if we collide with a particular object (such as a car). What is important is that there are no collisions with any "moving blobs". This approach also seems to be consistent with the early stages of motion processing in biological visual'systems [11]

We focus on the gradient-based techniques since they best correspond to the concept of motion as a primitive quantity and allow simple fusion of range and visual data. By applying a series of assumptions we transform the original twodimensional OFCE problem to a single dimensional problem leading to a significant reduction in computational complexity. We then develop our algorithm and present simulation results for our algorithm before discussing our proposed hardware implementation.

\section{MOTION Estimation Algorithm}

In this section we present the rational behind our algorithm. Beginning with the OFCE and the equations of motion a motion estimate for each pixel is calculated. Using a set of assumptions this $2 \mathrm{D}$ motion estimate is reduced to a single dimension then the resulting data is used to detect motion discontinuities hence segmenting the environment in terms of moving blobs.

\section{A. Optical Flow}

We begin with the optical flow constraint equation (OFCE - Eq 1), which relates image intensity derivatives to apparent image motion [5]. In this equation, $u$ and $v^{1}$ are the horizontal and vertical components of the apparent motion, and $I_{x}, I_{y}, I_{t}$ are the horizontal, vertical and temporal derivatives of image intensity. This equation is based on the assumption that, in the 2D projection of a scene, the brightness of a point on an object does not change with time. Thus all changes in the "brightness pattern" or image are due to motion. By applying

\footnotetext{
'In this presentation, bold italic variables are those unknown parameters for which we are seeking a solution. Measured values appear in a plain typeface.
} 
this equation to each pixel in the image we obtain a vector field that is an estimate of apparent motion throughout the image. This vector field is commonly known as optical flow.

$$
u_{(x, y)} I_{x_{(x, y)}}+v_{(x, y)} I_{y_{(x, y)}}+I_{t_{(x, y)}}=0
$$

Because the OFCE is a single equation in two unknowns, it cannot be solved directly and one is forced to make some assumptions regarding the nature of the solution. Typically, objects are assumed to move rigidly, hence neighboring image points move with approximately the same velocity.

\section{B. Motion Estimation}

Taking the equations of motion (under perspective projection) from [12] as our starting point and assuming ground plane motion, we can write the relationship between object velocity and apparent motion on image plane

$$
\begin{gathered}
\boldsymbol{u}=\psi\left(-\frac{1}{\mathrm{Z}}\left(\mathrm{x} \boldsymbol{T}_{Z}+f \boldsymbol{T}_{X}\right)\right) \\
v=\psi\left(-\frac{\mathrm{y} \boldsymbol{T}_{Z}}{\mathrm{Z}}\right)
\end{gathered}
$$

Substituting Eq 2 into Eq 1 gives our fusion between visual and range data [13].

$$
\mathrm{E}_{\mathrm{d}}=\psi\left(-\frac{1}{\mathrm{Z}}\left(\mathrm{x} T_{z}+f T_{x}\right)\right) \mathrm{I}_{\mathrm{x}}+\psi\left(-\frac{\mathrm{y} T_{z}}{\mathrm{Z}}\right) \mathrm{I}_{\mathrm{y}}+\mathrm{I}_{\mathrm{t}}=0
$$

We simplify this further by assuming that $T_{Z}$ is zero and all motion is modeled as horizontal motion $T_{X}[13]$.

$$
E_{d(x, y)}=-\frac{f \Psi}{Z_{(x, y)}} U_{x(x, y)} I_{x(x, y)}+I_{t(x, y)}=0
$$

We have replaced $T_{X}$ with $U_{X}$ since our interpretation of this parameter is now different. Whereas $T_{X}$ is the physical velocity (in meters/sec) of a point on an object, $\boldsymbol{U}_{\boldsymbol{X}}$ is the apparent horizontal motion $u$ reweighted to allow for distance, frame rate and pixel size. The ratio $f / Z(x, y)$ corrects for depth ambiguity where a distant object appears to move more slowly than a nearby object moving with the same velocity.

We call $U_{X}$ the apparent velocity because it corresponds to the speed at which a point on an object appears to be moving left or right. In the special case where the camera is stationary and an object moves strictly along the $\mathrm{X}$-axis, the apparent velocity will in fact be a correct estimate of $\boldsymbol{T}_{\boldsymbol{X}}$ otherwise it relates to the "rate of expansion" of the object. Since apparent velocity does not give a reliable measure of physical velocity, we only use it to segment the visual scene. Range information could then be used to assign a velocity to each segment.

To reduce quantity of data Eq 4 is solved at each pixel and the median motion estimate for each column is determined. Since our hardware provides a one dimensional laser scan we assume that depth is constant over the column. This is reasonable if the depth variation of object surfaces is small and images are relatively narrow (we use $512 * 32$ pixel images) such that only a single object is visible in any given column. The result is a robust estimate of motion for each column under the assumption that $U_{X}$ is approximately constant over each column.

\section{Smoothing and Segmentation}

Our next step is to segment the vector $U_{X}{ }^{2}$. We do this using a weak string constraint [14]. In this approach we imagine our final result as a string. The string should have a shape roughly similar to $U x$ however since all pieces of the string are connected, neighboring velocity estimates will have similar values. Because the string is weak, it may also break if it is stretched to far and these breaks form our segmentation. The concept of a weak string is captured in the following energy function that must be minimised in order to find a solution for the string.

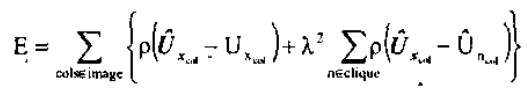

The first term of the summation constrains the smoothed value $\hat{U}_{x}$ to be relatively close to our estimate $U x$ and the second term constrains neighboring values of $\hat{U}_{x}$ to be similar effectively smoothing our data. The degree of smoothing is controlled by the $\lambda^{2}$ parameter. In this work we use the first order neighbors $U_{x c o l-1}$ and $U_{x c o i+1}$ as our clique. An appropriate choice for the estimator function $\rho(x)$ allows the string to break. Traditionally, $\rho(x)=x^{2}$ which leads to a least squares problem that is straight forward to solve however this does not allow the string to break. Instead we use a robust estimator functions such as the truncated quadratic [14] or Lorentzian [15] to allow segmentation. A side effect of using a robust estimator is that the resulting minimisation problem is non convex. To overcome this we use an approach known as graduated non-convexity (GNC) [14]. GNC is a continuation method where a set of approximations to the target estimator function is constructed under the control of a continuation parameter. Initially the continuation parameter is set to give a convex approximation and the resulting function is minimised. The continuation parameter is then updated and the new minimisation problem is solved using the previous result as a starting point. We continue updating the continuation parameter until the target estimator function is reached. GNC is ideal for hardware implementation since it is deterministic unlike other stochastic minimisation procedures.

\footnotetext{
${ }^{2}$ Because Ux has now been computed, it is no longer an unknown and we do not use bold italics.
} 

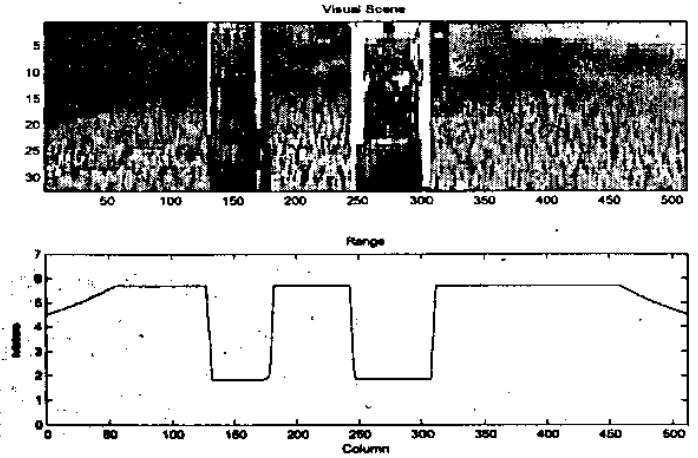

Figure 1. Example data
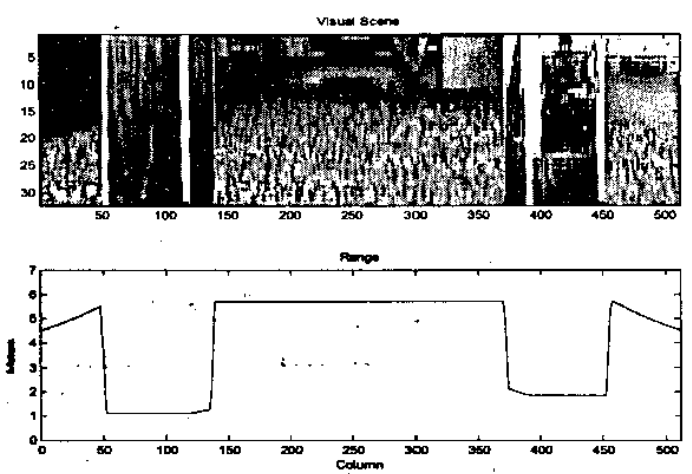

Figure 2. Example data

\section{AlgORITHM SimUlation RESUltS}

\section{A. Simulation System}

In order to verify and tune our algorithm we have developed a simulation system. This system is based on MATLAB and the freeware ray tracer Polyray. It generates both visual and range data for use with our algorithm, as well as correct apparent motion and physical velocity for testing purposes. The system allows the user to specify a simple virtual environment including the intrinsic and extrinsic camera parameters, camera motion, range finder parameters, lighting and the texture and motion of objects. Using this system we have created a database of scenarios that are similar to our laboratory environment. Figures 1 and 2 show typical data generated by the simulation system. The upper section shows the visual scene (stretched vertically) and the lower section shows the range data interpolated to match the cameras angular resolution.

\section{B. Simulation Results}

In this section we test an implementation of our algorithm where only 10 iterations of the minimization algorithm are used when segmenting the data and parameters remain unchanged between experiments. A single pair of frames is processed for each test. We first test our algorithm using the scenario depicted in figure 3 . Here the camera is stationary while two objects move through the environment.

The result is shown in figure 4 . The upper plot shows the

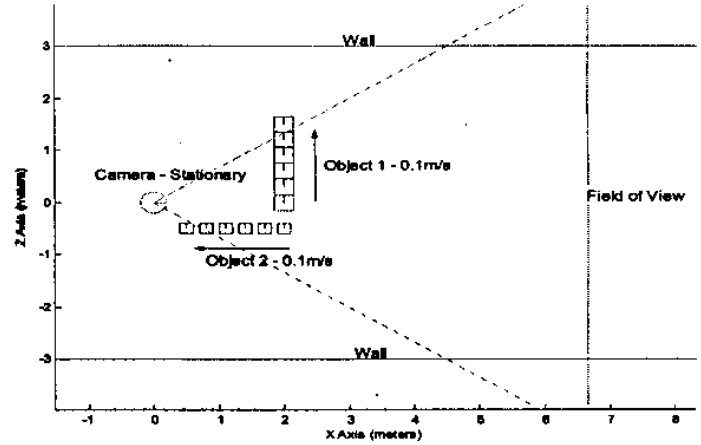

Figure 3. Scenario 1

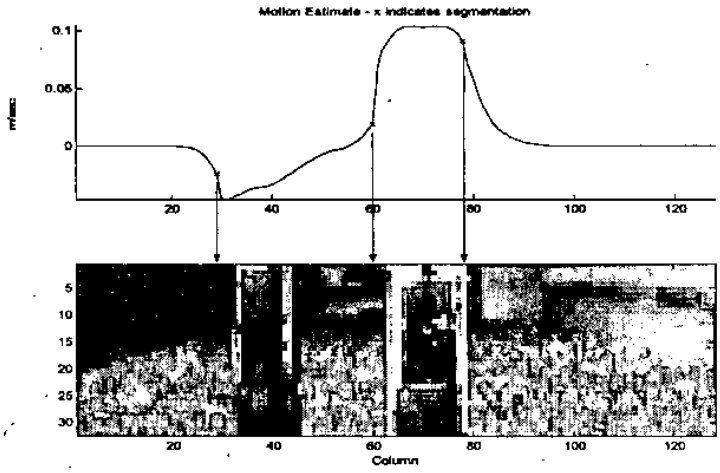

Figure 4. Result for scenario 1

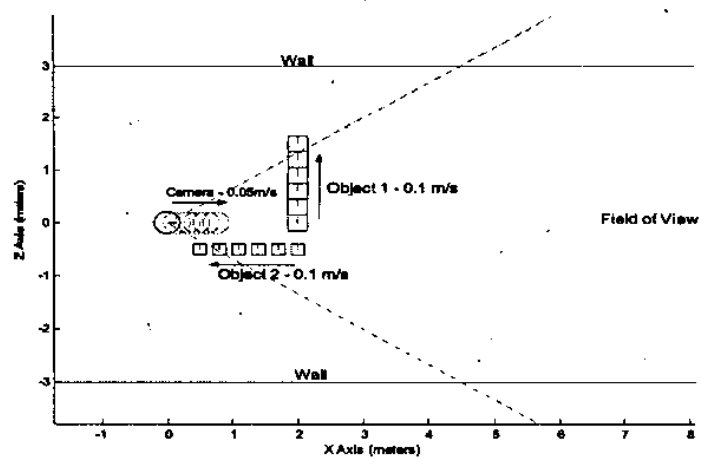

Figure 5. Scenario 2

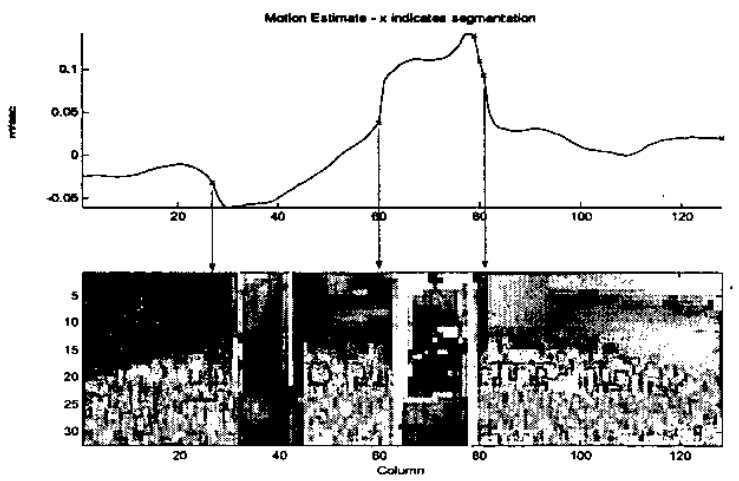

Figure 6. Result for scenario 2

estimated velocity at each column and crosses indicate locations where motion edges were detected. For reference, the visual scene is included in the lower part of the diagram. 


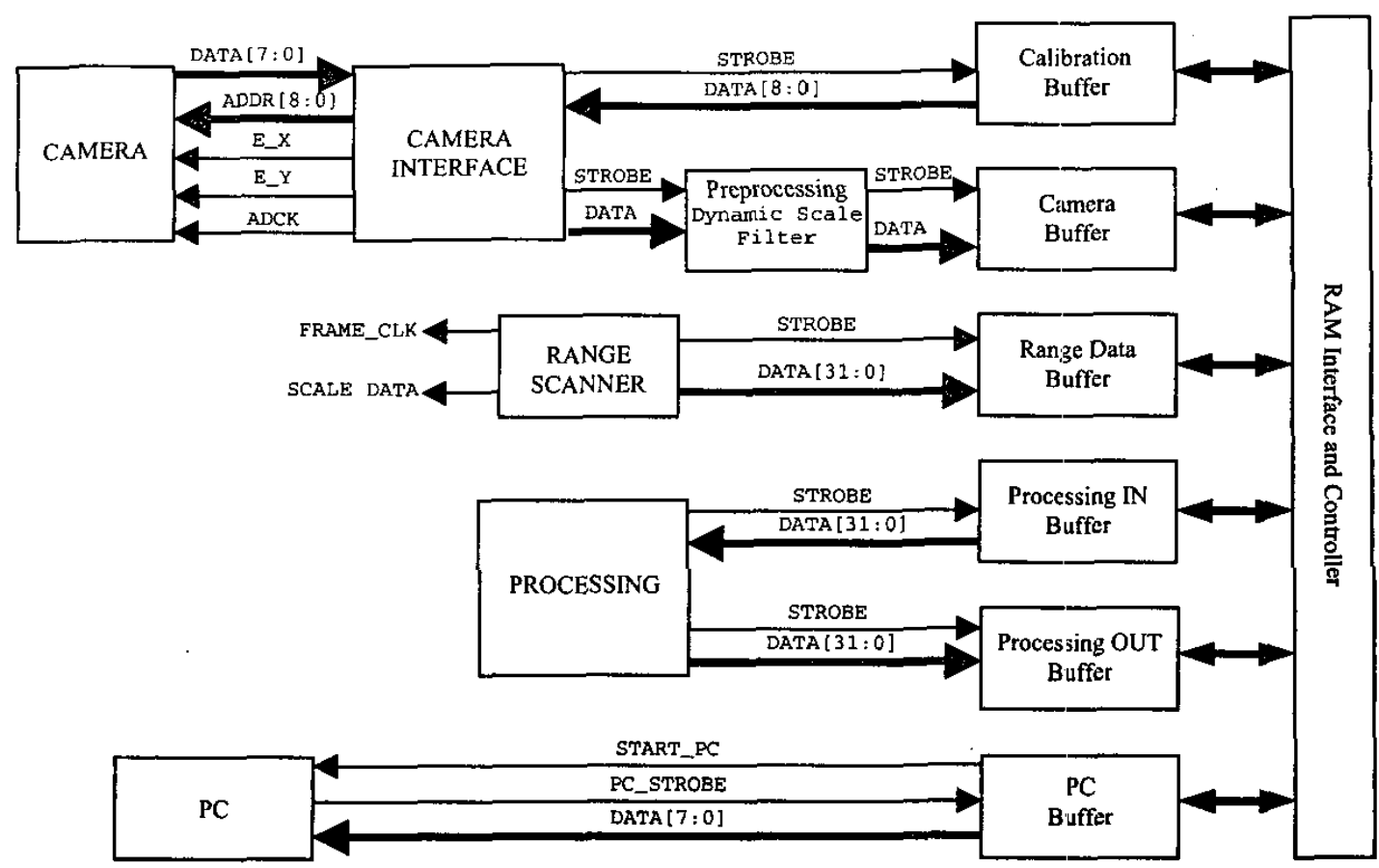

Figure 7. System Architecture

Our algorithm provides a good estimate of the absolute velocity of the object on the right, which is moving to the right at $0.1 \mathrm{~m} / \mathrm{s}$. Segmentation of this object has also been successful as indicated by the arrows. The left hand object has proven more difficult to segment - only the edge with the highest apparent motion has been detected. This failure has three causes. First and most critically, the apparent motion of this object is low, making it difficult to detect. This is exacerbated by the objects' lack of contrast, which leads to poor image derivatives and reduces the reliability of the resulting motion estimate. Finally, the first order weak string approach is only able to segment "step" discontinuities, hence the gentle change in velocity shown on the right hand side of object 2 cannot be detected.

Figure 5 illustrates our second scenario which is identical to the first except that the camera is now moving forward at $0.05 \mathrm{~m} / \mathrm{s}$. Since the camera is now in motion, it is no longer valid to equate the estimated apparent velocity to an objects physical velocity. Instead we focus on the estimated location of object boundaries. Once again we have been quite successful in locating the boundaries of object 1 though only one edge had been detected for object 2 . Once again, noise in the derivatives has lead to a shallow slope in the velocity estimate making the edge difficult to detect.

Notice the spurious discontinuities on the right of object 1. These are related to the peak above $0.1 \mathrm{~m} / \mathrm{s}$ in the apparent velocity. This peak corresponds to a peak in the underlying motion estimate that has not been adequately removed by a break in the data term. The result is an increase in the slope of the apparent motion curve in this region, which increases the likelihood of an edge being detected. This likelihood is increased further by a property of the weak string where double edges are formed if the slope is in a particular range [14].

Notice that for both experiments the localization accuracy is somewhat limited, especially for object 2 . This is caused by the low apparent motion of this object combined with the high degree of smoothing required. Tuning of parameters may improve localization however it is likely to increase the number of spurious discontinuities.

\section{IMPLEMENTATION DETAILS}

Figure 7 illustrates the architecture of our implementation. To allow for testing, a link to a PC allows for visualization of results though this link can easily be replaced by another device if further processing is required. This design consists of three key sections; the memory subsystem, a collection of processes (i.e. reading from camera, processing data, output to $\mathrm{PC}$ etc) and buffers designed to mediate communication between processes and memory.

\section{A. RAM Interface and Memory Management}

Because this system consists of a number of processes, each of which could require simultaneous access to RAM, bus arbitration is a necessity. Further, careful memory management (RAM allocation) leads to more efficient RAM use since devices use RAM differently.

Bus arbitration is implemented via a combination of decoupling buffers and the RAM Interface and Controller (RAMIC) module. Each process has a decoupling buffer implemented using Block SelectRAM (this RAM is within the FPGA so buffering does not cause further contention). The buffers allow each process to operate at full speed without waiting on memory. This is especially critical for the FUGA 


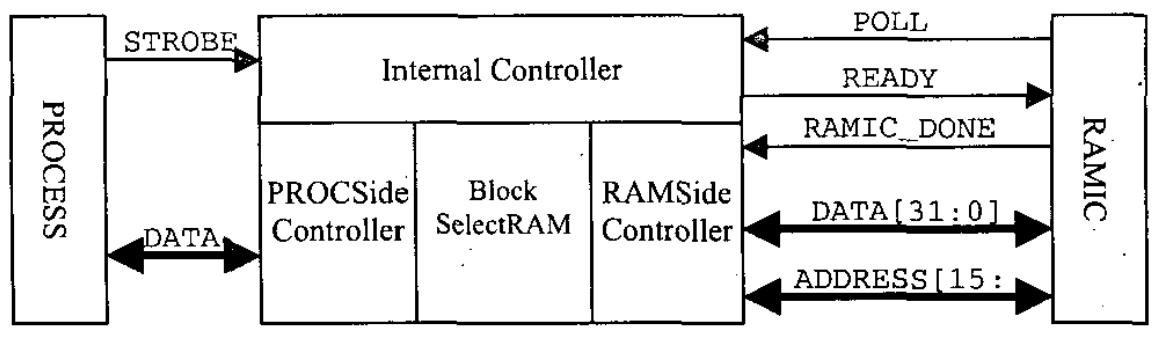

Figure 8. Structure of a buffer

camera where we must maintain a constant pixel rate to avoid distortion. The RAMIC polls each buffer in a round robin fashion allowing each buffer appropriate access to RAM and placing an absolute upper bound on RAM access times. To maintain a constant pixel rate, buffers do not pause when an overflow/underrun occurs. In this situation it is important to know the upperbound on memory access time so that the design can be made overflow/underun free. Aside from providing a low level interface to SDRAM, address decoding and bus arbitration, the RAMIC also provides a number of composite operations that allow more efficient RAM use. For example, we exploit the fact that data from the Fuga camera is 8 bits wide, while each SDRAM location is 32 bits wide to optimize memory access. A section of RAM is allocated as a "frame-buffer" with a one to one mapping from camera to RAM addresses, however we stack pixels so that each RAM location stores four frames of data. To implement this, existing pixel data is read from RAM, shifted 8 bits to the left and the new pixel is inserted in the least significant eight bits. From the point of view of the buffer, this is a single "write pixel" operation, which is more efficient than using the polling process to perform the implied read and write separately. However the real efficiency is realized when data is read for processing. Our algorithm requires 3 frames of data at each location to compute temporal image derivatives. With our pixel stacking scheme, this data can be obtained with a single RAM read operation.

The final function of the RAMIC is to generate zero-order calibration data for the FUGA camera. When the system is first started, the RAMIC generates a single image by taking the pixel-wise average of 16 frames of data. During this time a plain, translucent sheet of paper covers the lens so that the image seen by the camera contains only noise. Next, the distribution of pixel intensities is shifted so that is has zero mean. The resulting image represents the noise at each pixel called the calibration value. The corresponding calibration value is subtracted from each pixel to eliminate the fixed noise pattem.

\section{B. Buffers}

The Virtex FPGA has dedicated RAM (known as Block SelectRAM) available on. board. SelectRAM provides 4096 bits of storage, is dual ported, and the data width of each port can be chosen independently. A total of 28 blocks of SelectRAM are available on our XVC800 Virtex device.
Because we use SelectRAM for decoupling buffers we are able to implement buffers that can simultaneously be accessed by a process and by the RAMIC.

The specific design of buffers varies depending on their task though they all have the general structure shown in figure 8. The RAMSide controller mediates operations between SelectRAM and the RAMIC. Data and address lines between Buffers and the RAMIC are under tristate control. The handshake between a buffer and the RAMIC begins when the RAMIC sets a buffers polls line high. If that buffer has an operation pending, the RAMSide controller will assert the READY line and take control of the busses. The RAMIC now begins the requested operation and will assert the RAMIC_READY line when the operation is complete. Note that only a single operation is performed during a single poll, though this can be a compound operation' (such a stacking a pixel into RAM).

The PROCSide controller provides access to the buffer to a single process. Because the time to access the buffer is known these controllers do not use a two phase handshake like the RAMSide controller. Instead, the processes simply STROBEs the controller when an operation is required and the operation is completed one clock cycle later.

The intemal controller is a logical device whose implementation is spread across both the RAMSide and PROCSide buffers. The primary purpose of this device is to maintain buffer pointers and generate RAM addresses. In some buffers, this controller also performs some simple processing on the data stream.

\section{Camera Datapath}

In the camera data-path the Camera Interface generates the control signals necessary to read a $512 * 32$ pixel image from the camera. E_X and $E_{-} Y$ are the $X$ and $Y$ address strobes respectively, $A D D R$ contains the address and $A D C K$ (active low) is the analogue to digital conversion strobe and is controlled by a $1 \mathrm{MHz}$ pixel clock. Frame timing is controlled by the $10 \mathrm{~Hz}$ FRAME_CLK signal from the Range Scanner Interface. The camera interface also applies zero-order calibration to the raw camera data. The preprocessing block places image data into a queue (as per [16]) where a low pass filter is applied and the result is passed to the camera buffer. Data is written into RAM sequentially from address 0 up to address $(512 * 32)-1$ in row major order. 


\section{Range Scanner}

The range scanner block implements the interface to a range scanning device and provides support operations such as mapping the range data onto the camera coordinate system, and provision a frame clock and of scale data. Our target range scanner operates at $10 \mathrm{~Hz}$ and this rate will be used as the frame clock. This is slightly slower than our real-time rate of $12 \mathrm{~Hz}$ however $10 \mathrm{~Hz}$ is acceptable since, in practice, we will rarely experience the worst case conditions assumed in our temporal aliasing calculations. Scale data relates to the image width at the current scale and other related parameters.

\section{E. Processing}

Our system is designed so that the reading of new image and range data occurs concurrently with the output of data to PC. When the buffering of this data is complete, processing begins. The ProcessingIN buffer computes image derivatives and these are used by the processing block to generating a robust estimate of motion for each image column. This onedimensional motion estimate is then smoothed and segmented using a weak string model to produce the final motion estimate and segmentation.

\section{F. Output Process}

Because both the output of existing data to the PC and input of new from the camera and range finder occur concurrently, care must be taken to ensure data is not clobbered. We achieve this by ensuring that output to the PC always leads input of new data so that data is only updated once it has been output. To prevent possible buffer underruns, the PC Buffer reads ahead before triggering the $\mathrm{PC}$ to start accepting data (via the START_PC line). The PC (or other downstream device such as a DSP) is able to read a new value from the buffer by toggling the PC_STROBE line.

\section{G. Timing and Resource Use}

Our current implementation is complete except for the processing block and range scanner interface. This implementation has utilized approximately $15 \%$ of available logic resources, 35 digital IO's and 8 blocks of SelectRAM. Our implementation allows IO operations for the camera, PC and the laser range finder to be interleaved, however data processing must occur independently. Since I/O operations require $22.5 \mathrm{~ms}$ per frame, we have $77.5 \mathrm{~ms}$ available for processing giving a clock cycle budget of 19 clocks per pixel per iteration under the assumption of $512 * 32$ pixel images and 10 iterations per frame. This is more than sufficient for our algorithm.

\section{CONCLUSIONS}

In this paper we have introduced a revised approach to Horn and Schunks' optical flow algorithm where range information is used to disambiguate visual data and motion estimation is separated from motion segmentation to improve algorithmic efficiency. Simulation results indicate that our algorithm operates quite well, though some fine-tuning of parameters may be necessary. Finally we introduced the hardware architecture we use to implement this algorithm in real time. The final version of this paper will include practical results from the hardware platform.

\section{ACKNOWLEDGEMENTS}

We would like to thank Frauenhofer Autonomous Intelligent Systems Group (AiS) for donating of the Signal Master platform and Fuga Camera used in this work.

\section{REFERENCES}

[1] Barron, J. L., Fleet, D. J., Beauchemin, S.S., Systems and Experiment: Performance of Optical Flow Techniques, International Journal of Computer Vision, Vol 12, No 1, pp 43-77, February 1994.

[2] Kuhn P. M., "Fast MPEG-4 Motion Estimation: Processor Based and Flexible VLSI Implementations", Journal of VLSI Signal Processing, Vol 23, pp67-92, 1999

[3] Wiles, C. S., "Closing the loop on multiple motions", Ph.D. Thesis, Dept. Engineering Science, University of Oxford, Michaelmas, 1995

[4] Fleet D. J., Jepson A. D., 1990, "Computation of component image velocity from local phase information", Int J. Comp. Vis. Vol 5, pp77-104, 1990

[5] Horn B., Schunk, "Determining Optical Flow", MIT Artificial Intelligence Lab, AI Memo 572, April 1980

[6] Negahdaripour S., "Revised Definition of Optical Flow: Integration of Radiometric and Geometric Cues for Dynamic Scene Analysis", IEEE Trans on Pattern Analysis and Machine Intelligence, Vol 20(9), pp961-979,1998

[7] Heeger D. J., Hager G., "Egomotion and the Stabilized World", $2^{\text {nd }}$ Int. Conf Computer Vision, pp 435-440 1998

[8] Spies H., Jahne B., Barron J. L., "Dense Range Flow from Depth and Intensity Data", International Conference on Pattern Recognition, pp 131-134, 2000

[9] Sridhar B., Suorsa R., "Comparison of Motion and Stereo Methods In Passive Ranging Systems", IEEE Trans. Aerospace and Electronic Systems, Vol 27(4), pp 741-746, July 1991

[10] Nygards J., Wenersson A., "Model Based Fusion of Laser and Camera: Range Discontinuities and Motion Consistency", Proc. $3^{\text {rd }}$ Int. Conf. Information Fusion, vol 1, pp TUB5/16-21 2000

[11] Crick F., The Astonishing Hypothesis, Touchstone Books, Simon \& Schuster Ltd, London, 1995

[12] Mitiche A., "Computational Analysis of Visual Motion", New York, Plenum Press, ISBN: 0-306-44786-X, 1994

[13] Kolodko J., Vlacic L., "From Motion Processing to Autonomous Navigation", 2002 FIRA Robot World Congress, May 27-29, 2002

[14] A. Blake, A. Zisserman., "Visual Reconstruction", MIT Press, 1987, ISBN 0-262-02271-0

[15] Black M. J., "Robust incremental Optical Flow", Ph.D. Thesis, Yale, 1992.

[16] Röwekamp T., "A Smart Sensor System for Real-Time Optical Flow Estimation", Ph.D. Thesis, Technischen Universität Cottbus, 1997. 